\title{
ASPECTS QUANTITATIFS * DES EFFETS BIOCHIMIQUES DE L'IRRADIATION
}

\author{
G. MARBLÉ ** \\ Département de la Protection Sanitaire \\ Centre d'Etudes Nucléaires de Fontenay-aux-Roses
}

\section{INTRODUCTION}

L'action des rayonnements sur les organismes vivants consiste, initialement, en un transfert de l'énergie des rayonnements aux structures cellulaires. A cette phase physique, au cours de laquelle se sont formés des radicaux libres, des molécules excitées ou ionisées succède une phase physicochimique et chimique caractérisée par les variations des propriétés des structures biologiques et, en tout premier lieu, par l'altération des perméabilités et par des modifications de la structure des molécules chimiques. Ces modifications se produisent en un temps très court nettement inférieur à la seconde.

Les lésions biochimiques apparaissent ensuite et peuvent étaler leur développement dans les heures qui suivent l'irradiation. Elles se traduisent, tout d'abord, par des modifications de l'activité des enzymes et par des perturbations dans la coordination de l'action de celles-ci.

On conçoit aisément que les enzymes agissant à tous les niveaux et catalysant les réactions de synthèse ou de dégradation des molécules organiques, toutes les voies métaboliques des protéines, des glucides, des lipides vont subir des altérations ou des déviations, lesquelles entraîneront parfois des modifications de structure chimique des molécules constitutives des milieux organiques.

L'organisme réagit contre les désordres produits par l'irradiation en mettant en œuvre des mécanismes physiologiques de régulation et en éliminant certains produits toxiques formés par des réactions de détoxication.

Nous n'allons pas passer en revue toutes les voies importantes du métabolisme des composés organiques et rechercher les variations de ces métabolismes après irradiation. Nous allons, plus simplement, essayer de rassembler les résultats publiés des recherches effectuées, sur le plan biochimique, chez l'animal et l'homme irradiés, en ne retenant que ceux pour lesquels une expression quantitative, liée

* NOTA. - Ce texte fait suite à ceux de M. T. BIOLA et R. LE GO, publiés dans le no 1 - 1966. L'ensemble a été présenté au cours d'une réunion scientifique tenue par la Société le 3 r mars 1966 sur le thème "Aspects quantitatifs des effets biologiques de l'irradiation ".

** Service de Santé des Armées. détaché au Commissariat à l’Energie Atomique. 
à l'exposition a pu être établie. Nous ferons état, bien sûr, de nos propres travaux réalisés au Laboratoire de Radiopathologie du Département de la Protection Sanitaire.

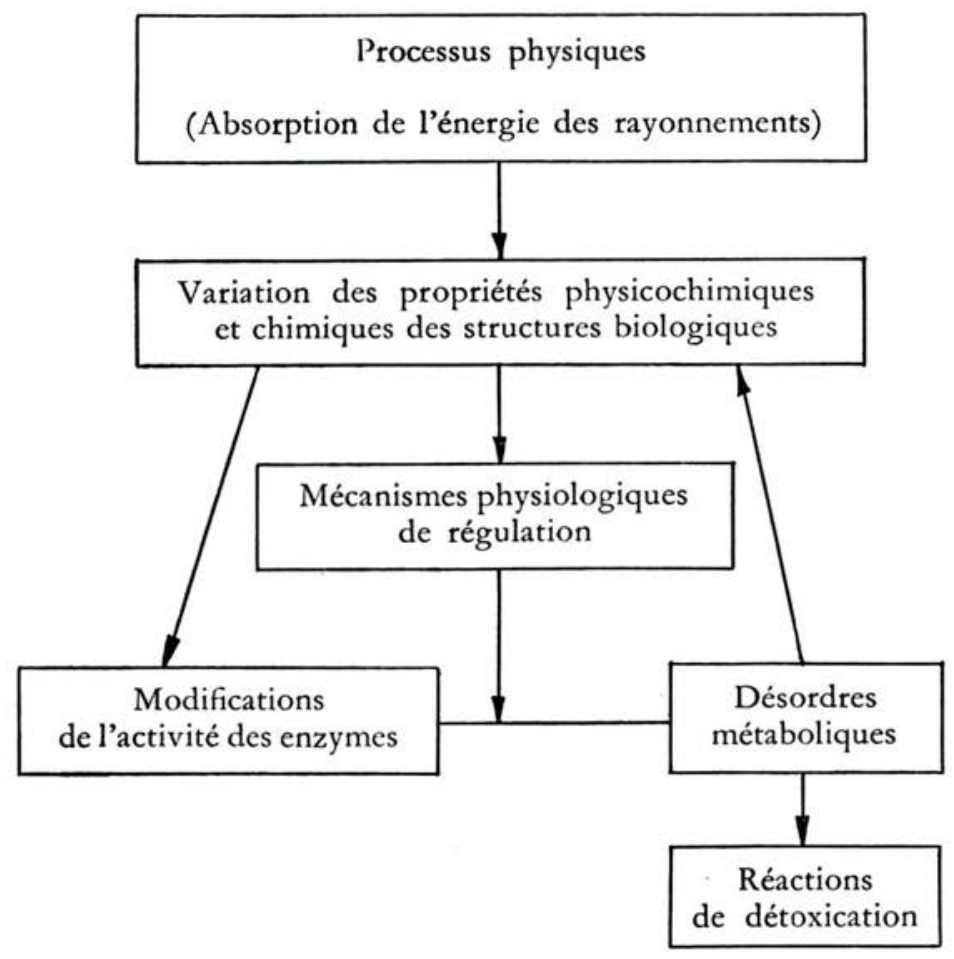

PRÉLÈVEMENTS

Les perturbations métaboliques peuvent être mises en évidence en réalisant le dosage chimique des produits intermédiaires des métabolismes dans des prélèvements effectués sur l'organisme irradié.

Les ponctions d'organes ou de tissus procurent un excellent matériel car on peut ainsi accéder au lieu même où se déroulent les réactions chimiques et contrôler directement l'activité des enzymes.

Les prélèvements de sang sont très utiles car le sang est le véhicule de transport des métabolites, et, soit dans le plasma, soit dans les globules, de nombreux systèmes enzymatiques participent à des réactions importantes, mais les variations sanguines des concentrations des composés chimiques, sont généralement assez faibles par suite de l'intervention des mécanismes physiologiques de régulation.

Le recueil des excrétas (fèces et urines) est indispensable, surtout l'urine par laquelle s'effectuent l'excrétion des catabolites d'élimination et des produits de détoxication. L'urine est, du point de vue biochimique, le prélèvement de choix par la spécificité des produits éliminés et la sensibilité avec laquelle peuvent 
être évalués les composés normaux ou anormaux décelables par l'analyse chimique et physicochimique.

\section{ACTIVITÉ ENZYMATIQUE}

Nous avons indiqué le rôle capital joué par les enzymes dans le déroulement et l'enchaînement des réactions chimiques du métabolisme normal. L'action de l'irradiation sur l'activité enzymatique est assez variable.

Certains enzymes tels que les transaminases et les deshydrogénases sériques, la leucine aminopeptidase et la phosphatase acide sériques sont le plus souvent activés (1 2, 13, 14, 15, 29, 35, 56) mais la réponse en fonction de la dose absorbée affecte diverses modalités.

Il semble que l'on ait, par exemple, pour la lactodeshydrogénase sérique, une réaction à seuil, l'élévation de l'activité enzymatique ne se produisant, chez le singe, qu'à partir de 300 rads et ne variant guère ensuite $(12,13)$.

Hietbrink, Raymund, Zins et Dubois ont trouvé une relation linéaire entre l'activité enzymatique et l'exposition pour l'adénosinetriphosphatase de la rate de rats irradiés et sacrifiés trois jours après l'irradiation (28), (cf. fig. I).

\section{Activité}

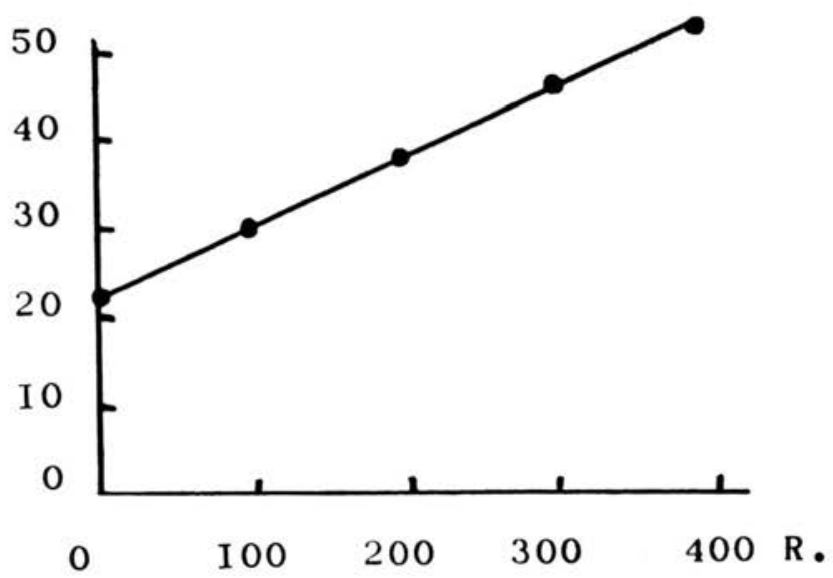

Fig. 1. - Activité de l'adénosine triphosphatase de la rate de rat.

Par contre, l'irradiation peut aussi provoquer une décroissance ou une inhibition temporaire de l'activité des enzymes. L'activité des aldolases, de la glycérophosphate isomérase décroît après irradiation. Il en est de même, de l'activité de la cholinestérase de l'intestin de rat, la variation étant fonction de la dose comme l'ont également montré les chercheurs de l'U.S.A.F. de Chicago (28), (cf. fig. 2). 
RAPPOPORT et Fritz (48) ont étudié l'inhibition de la nucléosidephosphorylase des érythrocytes. Cet enzyme est très important car il régit le métabolisme des nucléosides, en particulier celui de la guanosine, de l'inosine et de l'adénosine.

Activité

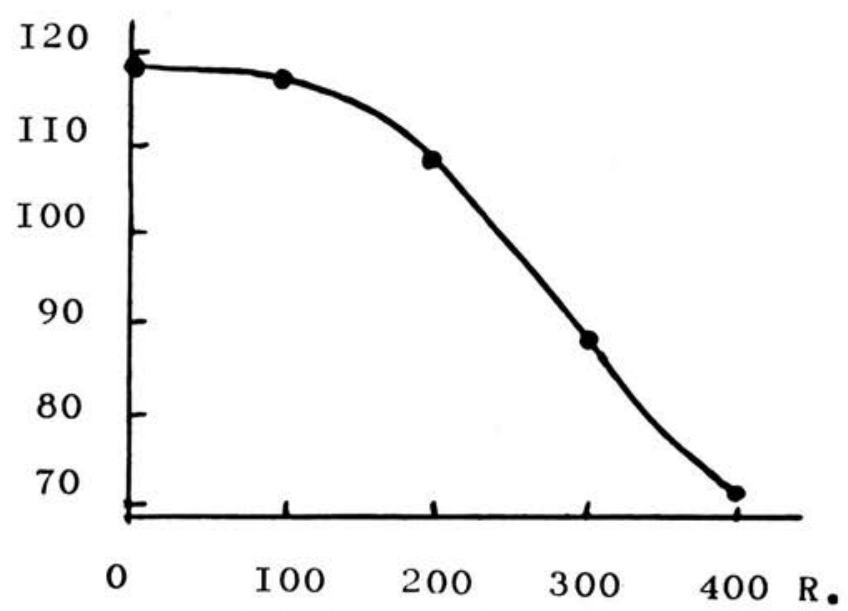

Fig. 2. - Activité de la cholinestérase de l'intestin du rat.

Les courbes de la figure 3 rendent compte de l'inhibition de l'enzyme en fonction de la dose reçue. Le retour à la normale s'effectue au bout d'un temps qui est proportionnel à l'exposition. La relation semble linéaire jusqu'à soo R. (cf. fig. 4).

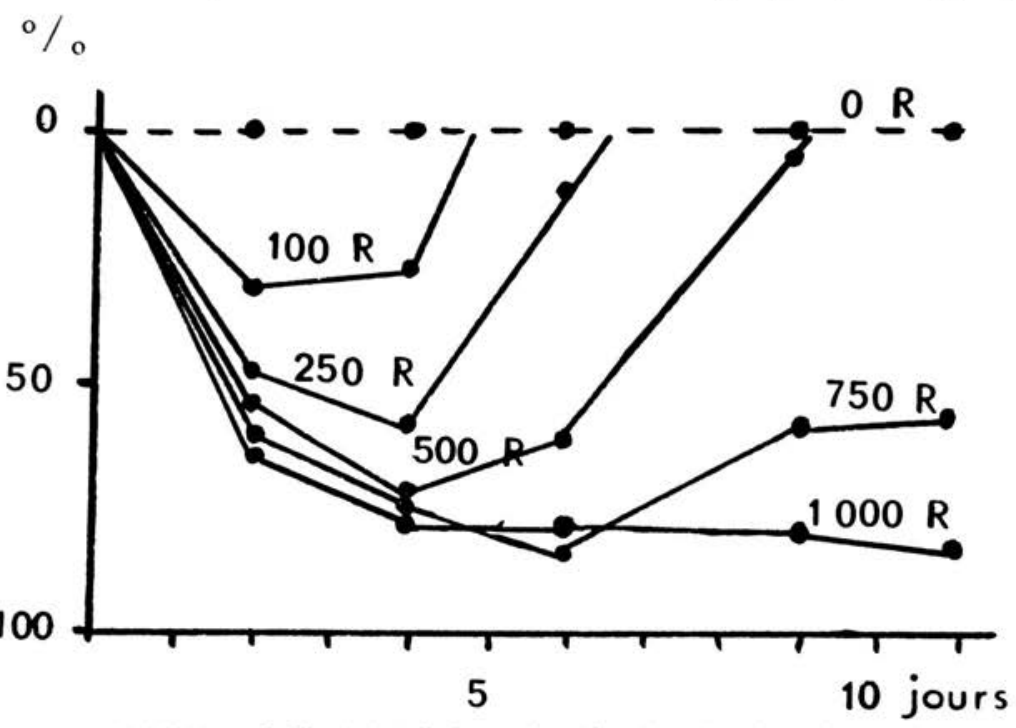

FIG. 3. - Inhibition de l'activité de la nucléoside phosphorylase des érythrocytes. 


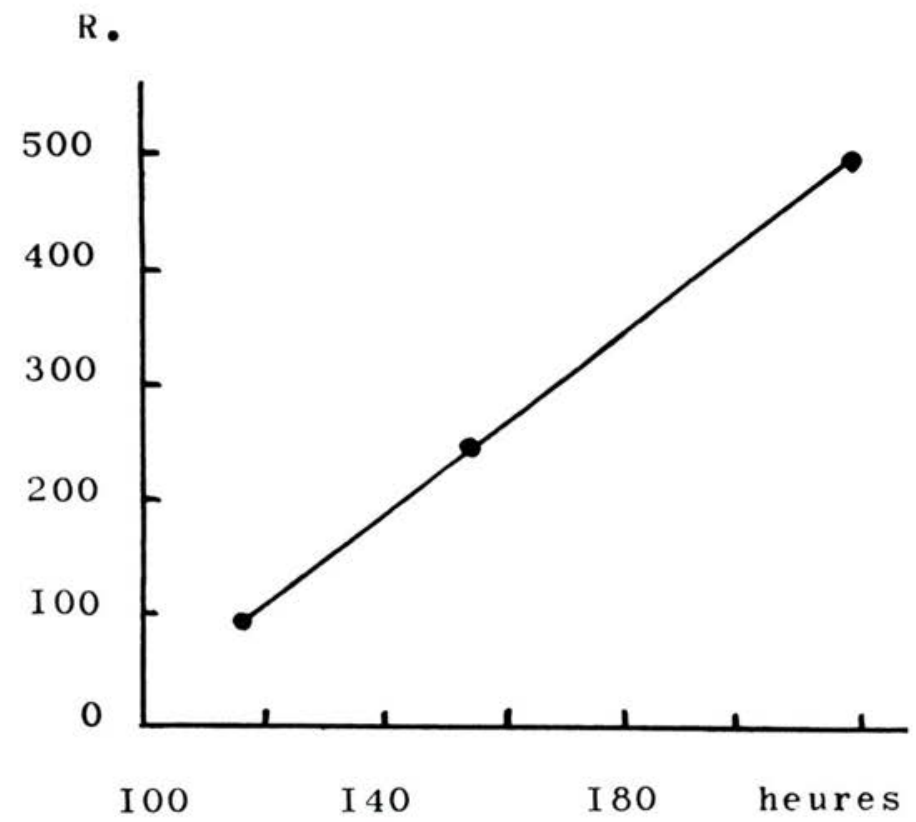

FIG. 4. - Relation Exposition-Durée d'inhibition de la nucléoside phosphorylase des érythrocytes.

\section{VARIATIONS SANGUINES}

Nous venons de voir un exemple de variation de l'activité d'un enzyme érythrocytaire. Les mesures d'activité enzymatique sont sans doute les tests les plus sensibles, au niveau du sang, car par suite de l'intervention des mécanismes régulateurs il ne se produit que de faibles variations sanguines aux doses sublétales. Il en est ainsi des concentrations plasmatiques d'électrolytes (40) ou des constituants protéiniques.

\section{Protéines sériques.}

A dose élevée, on note des variations des concentrations plasmatiques des fractions protéiniques du sang. On peut constater une augmentation relative des globulines $\alpha_{1}$ et $\alpha_{2}$ et une diminution relative des globulines $\gamma$, les protéines sanguines diminuant en valeur absolue. Mais comme le montrent les courbes établies par Winckler et PaschKe (62) (fig. 5), le rapport globulines $\alpha$ sur globulines $\gamma$ n'est nettement augmenté que pour des valeurs supérieures à $400 \mathrm{R}$.

\section{Autres composés.}

Une augmentation de l'acide urique et de l'azote non protidique ( $5 \mathrm{I}$ ), de la bilirubine (38), du cholestérol, de la créatine, ont été observées. Certaines variations plasmatiques sont dues, pour une grande part, à la variation de l'hémato- 
crite, c'est-à-dire du rapport globules/plasma qui peut varier soit par départ d'eau plasmatique, soit par diminution du nombre des globules.

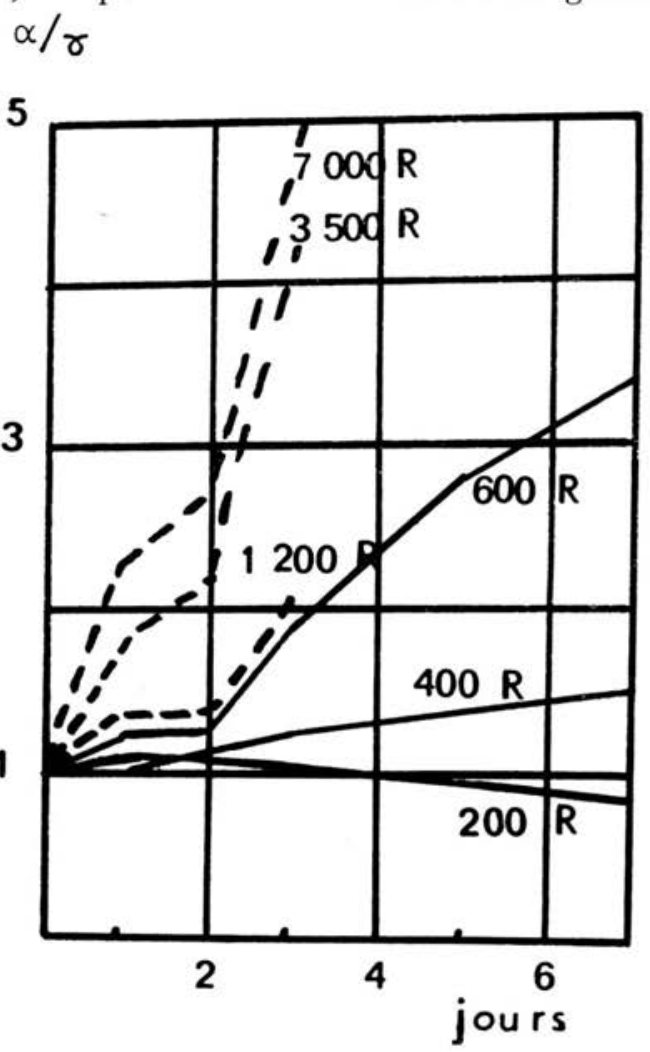

FIG. 5. - Rapport globulines $\alpha /$ globulines $\gamma$

\section{Glycémie.}

Il peut être intéressant de suivre, plus en détail, la variation de la glycémie car elle peut traduire l'action des rayonnements sur un mécanisme physiologique complexe de régulation.

On note, généralement, après l'irradiation une hyperglycémie dontl'amplitude et la durée est variable selon la dose mais aussi selon l'espèce à laquelle appartient l'animal irradié. A cette hyperglycémie fait suite une hypoglycémie, le retour éventuel à la normale pouvant s'effectuer après plusieurs oscillations. Nous avons observé ces variations chez l'homme (cf. fig. 6) et chez le rat (39) et il semble que l'on puisse tirer des conclusions relatives aux chances de survie de l'animal en étudiant l'amplitude, la période et le nombre des oscillations. Les valeurs extrêmes de l'hyper- ou de l'hypoglycémie sont difficiles à utiliser car elles ne sont pas toujours observées au même moment pour des doses différentes, comme le montrent les courbes des figures 7 et 8 relatives à des rats irradiés dans des conditions analogues mais non identiques. 
$g \cdot / 1$ 。

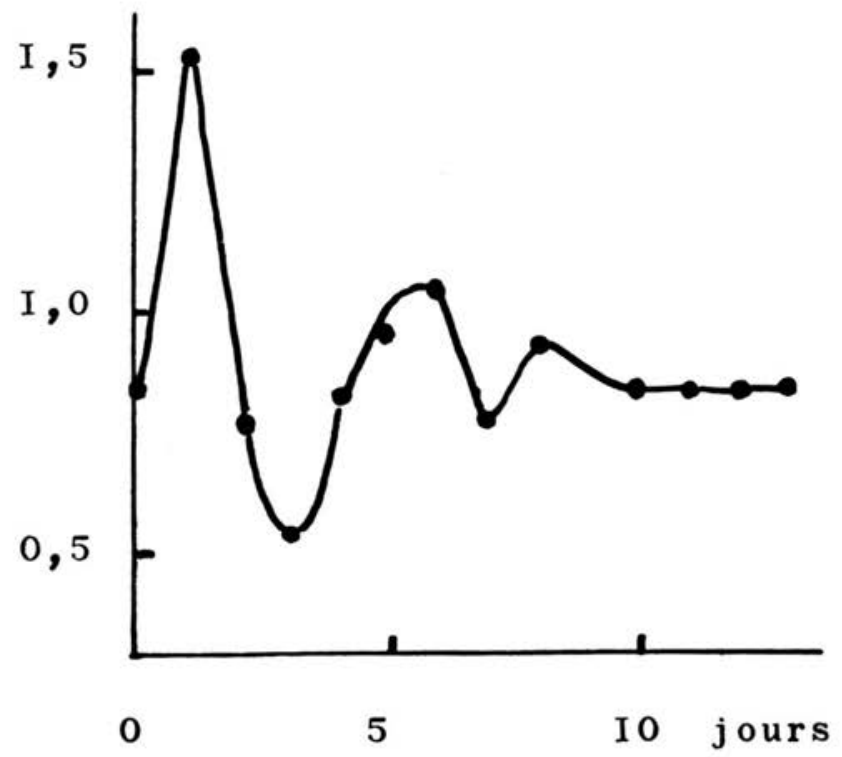

FiG. 6. - Variation de la glycémie chez l'homme irradié.

g. / I

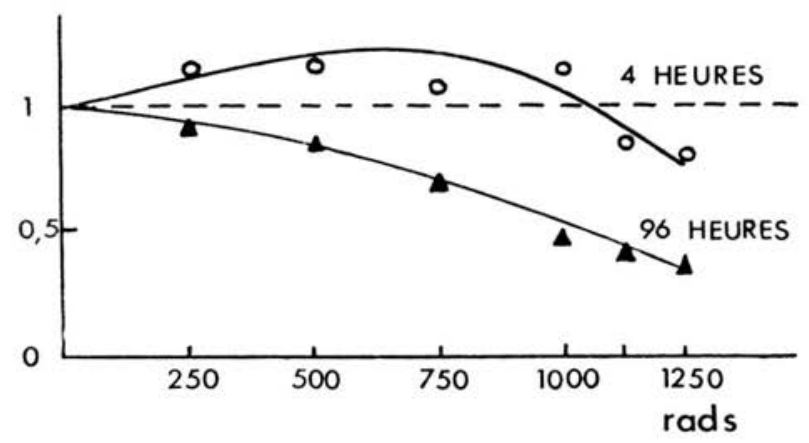

Fig. 7. - Variation de la glycémie chez le rat irradié.

Ces courbes mettent en évidence le caractère dynamique des variations de nature biochimique que l'on observe après irradiation et montrent qu'il est indispensable de mettre l'accent sur la notion de temps qui doit toujours être noté, avec précision, à côté de toute valeur expérimentale. 


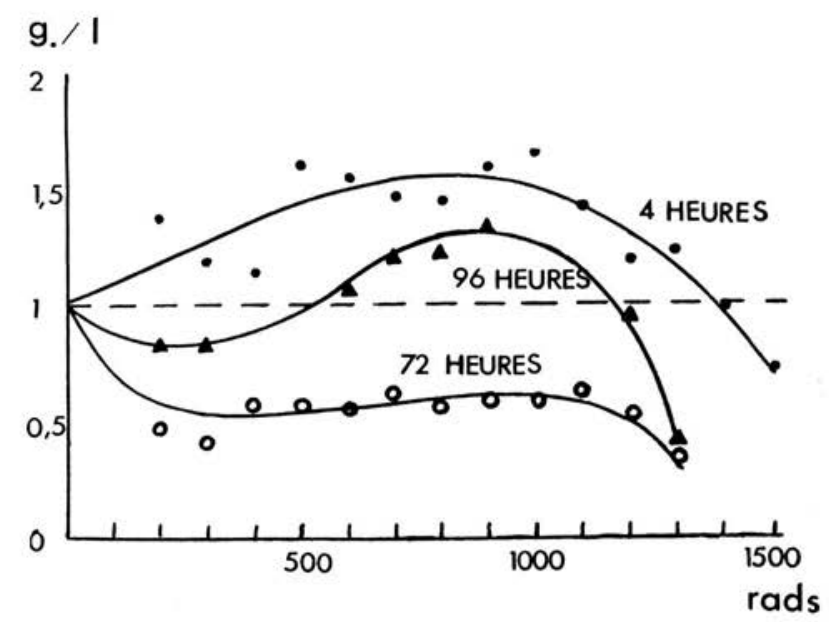

Fig. 8. - Variations de la glycémie chez le rat irradié.

EXCRÉTION URINAIRE

La notion de temps est, également, très importante en ce qui concerne les prélèvements urinaires. On recueille, généralement, les urines de 24 heures ou bien l'on détermine le rapport de la valeur de la concentration de l'élément recherché à celle de la créatinine qui semble peu touchée par l'irradiation et dont la quantité excrétée est fonction du volume d'urine émis. L'utilisation des courbes cumulées, en fonction du temps, présente un intérêt certain car elle rend compte de l'évolution des variations.

Les recherches ont le plus souvent porté sur le métabolisme des protéines, en particulier les acides nucléiques et les acides aminés, plus rarement sur les composés minéraux, les stéroïdes ou les catabolites des métabolismes lipidique et glucidique.

\section{Créatine.}

L'excrétion de créatine a été particulièrement étudiée par ANDERSON, GERBER, Altman, Hempelmann (20, 21, 22, 23, 24, 27, 61). Gerber a noté une élévation nette de la créatinurie du rat après irradiation, le rapport créatine/créatinine est proportionnel à la dose, tout au moins jusqu'à $700 \mathrm{R}, \mathrm{le}_{2} \mathrm{e}$ jour après l'irradiation et dans les urines des quatre premiers jours cumulés (fig. 9).

Les valeurs relativement plus faibles trouvées pour des doses supérieures à $700 \mathrm{R}$ trouvent leur explication dans le fait qu'il existe un décalage de deux jours environ dans l'apparition du maximum de l'excrétion correspondant à $2000 \mathrm{R}$ ( $c f$. fig. 1o). Un second maximum est d'ailleurs observable vers les $9^{\mathrm{e}}$ et $1 \mathrm{o}^{\mathrm{e}}$ jours.

La synthèse de la créatine reste normale, c'est l'utilisation par les muscles de la créatine formée qui est perturbée.

La créatinurie a été mesurée dans les urines des irradiés de Los Alamos et d'Oak Ridge (2, 21, 27). La créatinurie avait une valeur élevée chez tous les irradiés mais la corrélation excrétion/exposition n'a pas été parfaitement démontrée. 
Créatine/Créatinine

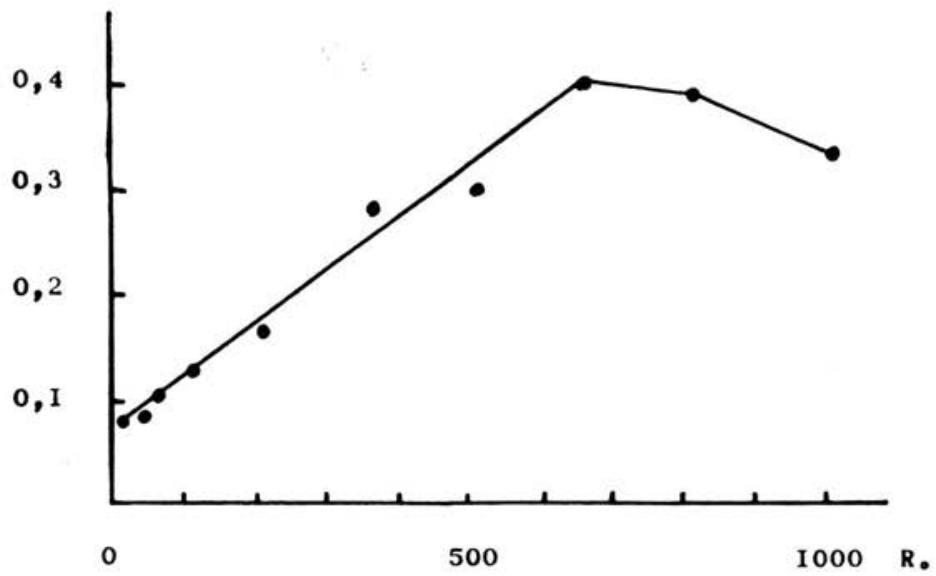

FIG. 9. - Créatinurie du rat après irradiation.

Créatine / Créatinine

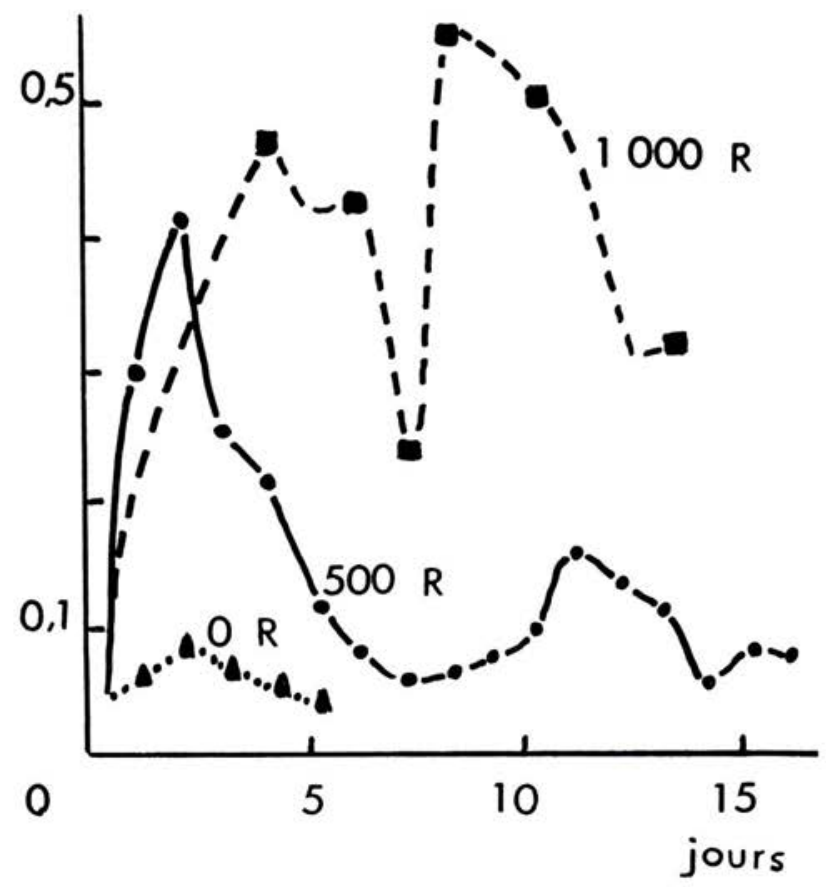

FIG. 10. - Rapport Créatine/Créatinine. 
Pseudo uridine.

La pseudo uridine libre est sans doute le produit final de la dégradation de l'acide pseudo uridylique contenu dans l'acide ribonucléique et traduit ainsi la dégradation de l'A.R.N. tissulaire. Drahovsky, Winkler et SKoDA (17) ont trouvé une relation linéaire entre l'élimination urinaire de pseudo uridine et l'exposition, le maximum de l'excrétion se situant le $\mathbf{2}^{\mathrm{e}}$ jour après l'irradiation (cf. fig. I I).

$\mathrm{mg}$ 。

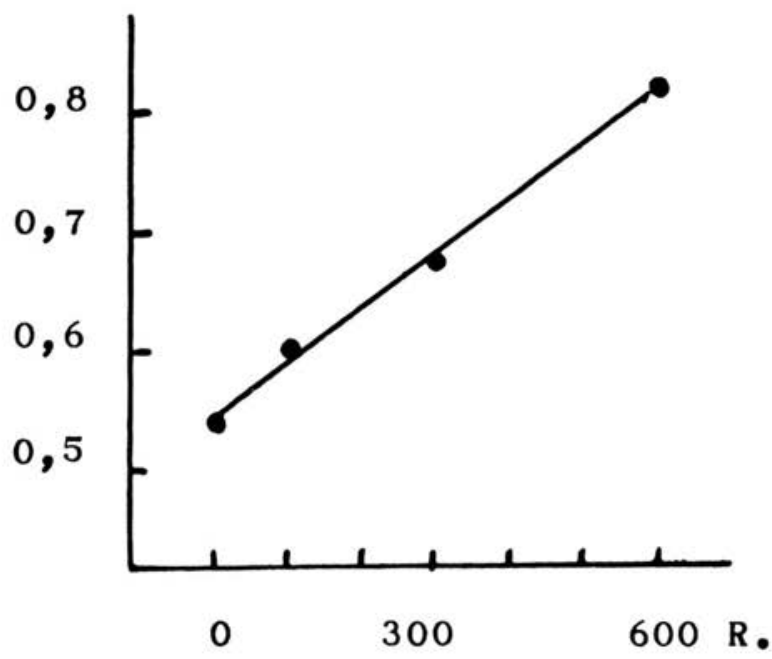

Fig. II. - Pseudo uridine urinaire en fonction de la dose.

\section{Desoxycytidine.}

L'excrétion de la désoxycytidine, produit de dégradation de l'acide désoxyribonucléique a été, également, trouvée augmentée dans les 24 heures qui suivent l'irradiation, dans des proportions fonctions de la dose reçue $(6,43)$.

\section{Acides aminés.}

L'augmentation de l'excrétion des acides aminés a été notée dans tous les accidents d'irradiation que ce soit à Los Alamos, à Oak Ridge ou chez les yougoslaves de Vinça $(2,27,30,44,46,5 \mathrm{I})$.

B.A.I.B.A.

L'acide aminé qui a été le plus étudié est l'acide $\beta$ aminoisobutyrique ou B.A.I.B.A., c'est un catabolite de la thymine et de thymidine. Son élimination urinaire augmente, sans aucun doute, dans les jours qui suivent l'irradiation mais la proportionnalité élimination/dose n'a pu jusqu'à présent être affirmée. SMrTH (ss), pour sa part, est convaincu que l'on ne peut employer l'excrétion du B.A.I. 
B.A. comme indicateur d'exposition aux radiations ionisantes. D'autres auteurs (43) ont retrouvé de la thymidine libre dans les urines d'irradiés alors que l'on n'en a pas retrouvé dans les urines de l'accidenté de Los Alamos, en 1958, qui avait reçu plus de 10000 rads $(5 \mathrm{I})$. Il est à noter que dans ce cas particulier une grande quantité de B.A.I.B.A. avait été excrétée dans les urines.

\section{Taurine.}

La taurine qui est un acide aminé soufré a également été très étudiée. Chez le rat, le maximum de l'excrétion se produit environ six heures après l'irradiation (cf. fig. 1 2).

Pour les accidentés d'Oak Ridge le maximum se situait à la fin de la première semaine.

Watson (6o) ainsi que Boquet et Fromageot (8) pensent que l'excrétion de la taurine serait due à la destruction du tissu lymphoïde et des cellules à index mitotique élevé.

$$
\text { mg / heure }
$$

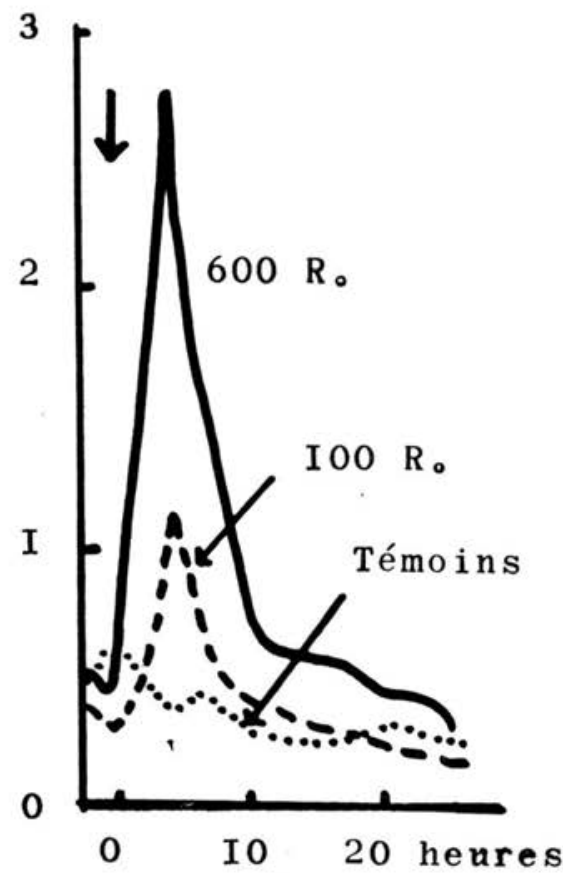

FIG. I 2. - Excrétion urinaire de taurine chez le rat irradié.

Acides $\alpha$ aminés.

En ce qui concerne les acides $\alpha$ aminés, on constate qu'une grande quantité de glycocolle provenant de la dégradation du collagène est libérée alors que l'excrétion de la sérine libre est diminuée $(2,27,30,44)$. Des aminoacides ne 
s'excrétant que très peu par l'urine, normalement, tels que la proline, la phénylalanine, l'acide aspartique, la thréonine, le tryptophane ont été retrouvés. Des études, en cours dans notre laboratoire, permettront peut-être de savoir si une relation dose/effet apparaît.

\section{Acides organiques.}

L'excrétion urinaire de certains acides organiques augmentent après l'irradiation, il en est ainsi de l'acide pyruvique, de l'acide $\alpha$ cétoglutarique, de l'acide lactique (\$2).

\section{Amines at 5-H.I.A.A.}

Il en est de même pour la plupart des amines, entre autres l'histamine, la tryptamine, la sérotonine ou s-hydroxytryptamine (18, 19) dont l'importance est à souligner parce qu'elle est, à l'heure actuelle, un des meilleurs radioprotecteurs chimiques connus et que son produit de dégradation l'acide s-hydroxyindoleacétique ou s-H.I.A.A. s'élimine par l'urine d'une façon sensiblement proportionnelle à la dose comme l'ont montré DeAnovic, Supek et RANDic $(15,47)$ (cf. fig. I 3 ).

$$
\mu g / \text { heure / I00 g. de poids }
$$

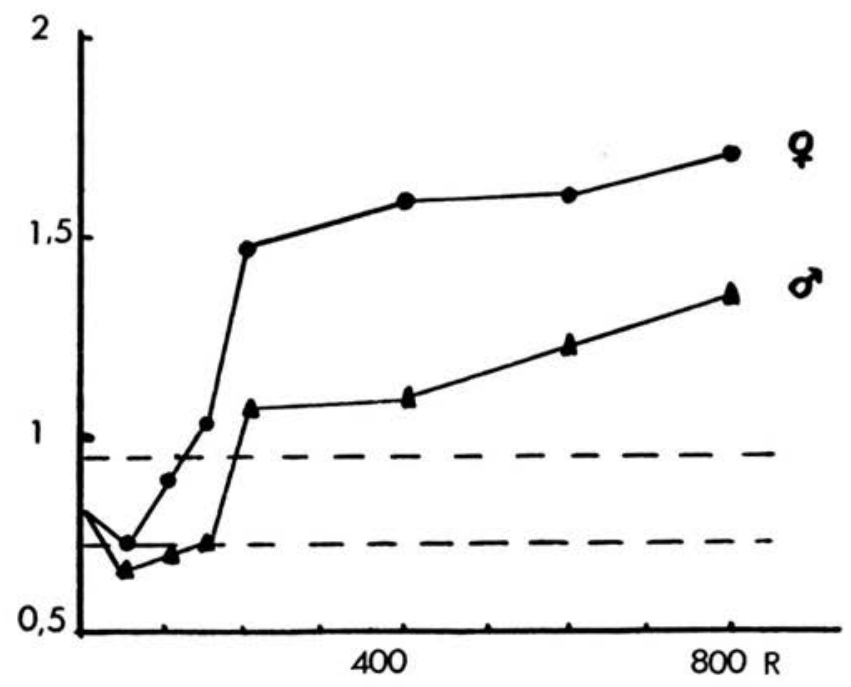

Fig. 13. - Excrétion urinaire de s-H.I.A.A. en fonction de la dose

\section{Electrolytes.}

L'excrétion des électrolytes est, en général, fonction des quantités ingérées cependant nous avons pu observer, chez le rat, que l'excrétion du chlore, du potassium, du sodium était d'autant plus faible que la dose reçue était forte surtout dans les premiers jours après l'irradiation comme le montrent les courbes 
$\mathrm{m} \mathrm{Eq}$

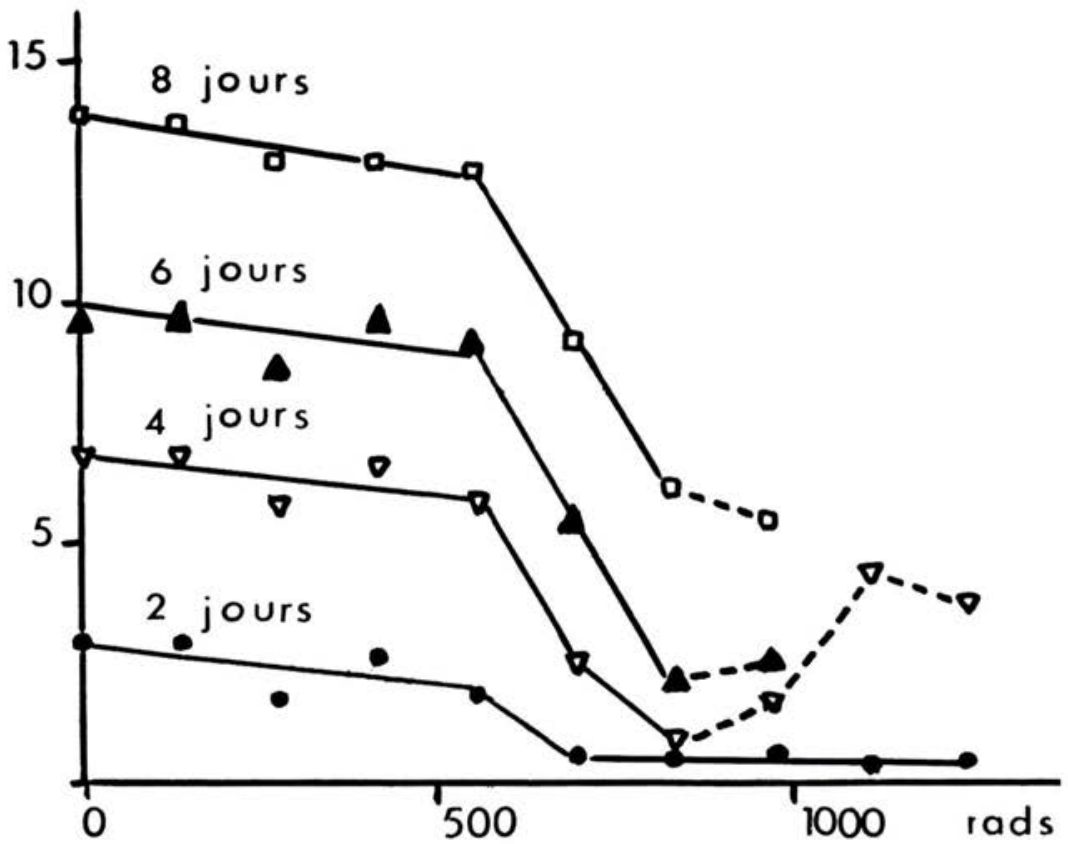

FIG. I4. - Excrétion urinaire de sodium chez le rat.

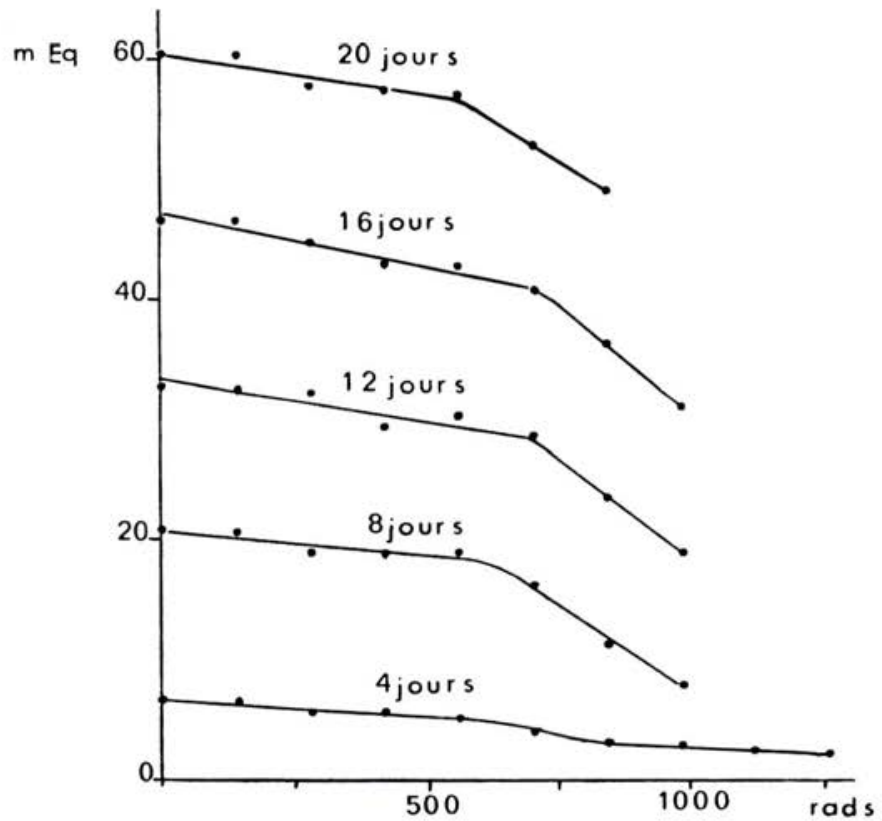

Fig. 15. - Excrétion urinaire de potassium chez le rat. 
des excrétions cumulées de sodium et de potassium représentées par les figures ${ }_{1} 4$ et I 5 . Pour les doses supraléthales ceci n'est, peut-être, plus vrai pour le chlore et le sodium mais il est difficile de l'affirmer car il est alors malaisé de séparer les urines des selles diarrhéiques.

\section{Glycuronides.}

Un exemple de processus de détoxication nous est donné par Scoppa et Gerbaulet dans un rapport Euratom très récent (53). Ces auteurs ont irradié des rats à $200,300,660$ et $880 \mathrm{R}$ et ont déterminé les quantités de glycuronides excrétés dans l'urine pendant les quatre premiers jours après l'irradiation ( $c f$. fig. 16).
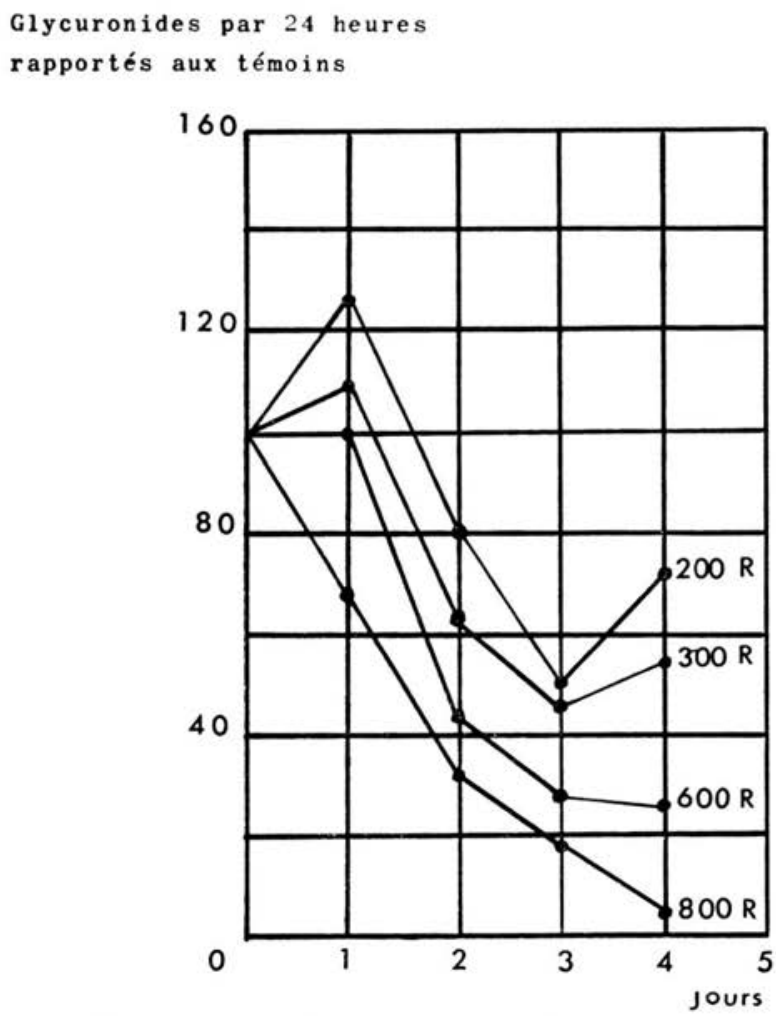

FIG. 16. - Excrétion urinaire des glycuronides.

PRÉLÈVEMENTS TISSULAIRES

La variation des concentrations tissulaires de certains composés chimiques peut, dans de nombreux cas, être proportionnelle à la dose, nous en avons vu des exemples concernant les enzymes. 
Skalka et Matyasova ( 54 ) ont dosé les désoxyribonucléotides libres dans la rate de souris irradiées et ils ont établi une relation linéaire avec l'exposition jusqu'à $300 \mathrm{R}$ avec une précision de $35 \mathrm{R}$ ( $c$. fig. 17). Ce dosage présente un intérêt certain dans la détermination des petites doses d'aurant plus que ces mêmes auteurs ont obtenu une relation analogue dans les prélèvements de moelle osseuse, prélèvements qui sont pratiquement de règle, dans le cas d'irradiation accidentelle, pour évaluer l'activité hématopoïétique de la moelle.
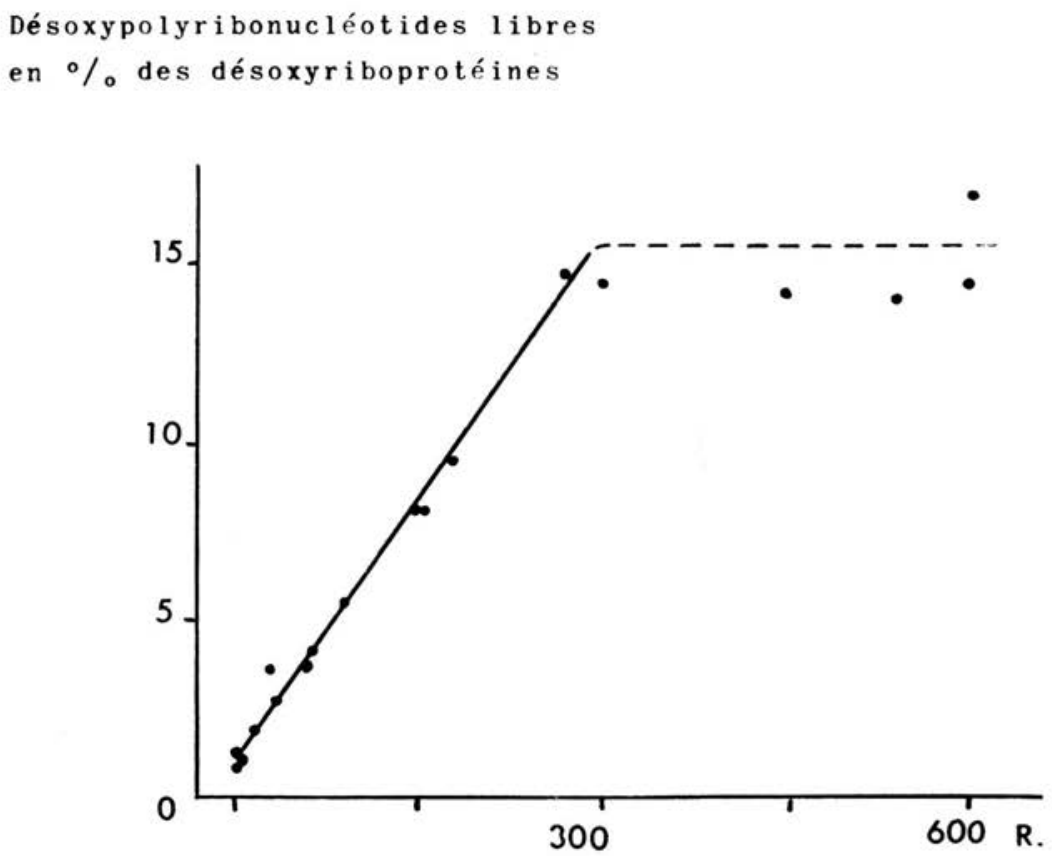

FIG. 17. - Désoxypolyribonucléotides libres de la rate de souris.

\section{EFFETS DES NEUTRONS}

Les effets biochimiques propres à l'action des neutrons ont été peu étudiés jusqu’à présent. Un fait a cependant été signalé par KRETCHMAR d'Oak Ridge (34). Cet auteur a constaté que le troisième jour après une irradiation due à un accident de criticité, c'est-à-dire gammas + neutrons, on ne retrouve plus de sérine libre dans les urines de l'accidenté, or en effectuant le même dosage sur des urines d'irradiés thérapeutiques donc gamma uniquement, la quantité de sérine libre excrétée le troisième jour est normale.

Quant à la dosimétrie biologique des neutrons elle est effectuée de façon courante, par les méthodes classiques d'activation (sodium du sang, soufre des phanères, etc.). Le résultat ne peut être qu'approximatif puisque la quantité d'éléments activés varie avec l'énergie des neutrons. 


\section{REMARQUES}

Avant de tirer la conclusion de ces résultats, il faut rappeler que les travaux dont ils sont l'expression ont, pour la plupart, été effectués chez l'animal. Les données humaines ne sont fournies que par les accidentés qui sont, heureusement, peu nombreux et les malades irradiés à des fins thérapeutiques. Dans ce dernier cas, les sujets sont, le plus souvent affaiblis par la maladie et les irradiations qui sont pratiquées sont, en général, partielles.

Il faut constater, également, que les variations observées sont, dans la majorité des cas, non spécifiques de l'irradiation. Cet inconvénient est, en fait, mineur si l'effet observé est nettement relié au dommage subi.

\section{CONCLUSION}

Nous concluerons en disant que les effets biochimiques de l'irradiation peuvent servir à l'évaluation quantitative du dommage biologique subi par l'irradié. Le seuil de sensibilité peut être situé à quelques rads, la précision ne pouvant atteindre qu'une cinquantaine de rads pour les meilleures méthodes. On peut donc réaliser une dosimétrie biochimique, chez l'animal, malheureusement l'extrapolation à l'homme des résultats expérimentaux n'est pas encore possible. L'intérêt des méthodes biologiques, en particulier biochimiques, réside dans le fait qu'elles seules peuvent permettre de définir la nature exacte des dégâts subis par les organismes irradiés et, par suite, le traitement qu'il y a lieu de mettre en œuvre pour que le développement des effets biologiques d'une irradiation sévère ne conduise inéluctablement à une issue fatale. On peut raisonnablement penser que, d'ici quelques années, les méthodes biochimiques associées, bien sûr, aux autres techniques biologiques telles que l'hématologie, sans oublier les précieux renseignements apportés par la dosimétrie physique, permettront d'évaluer, avec une précision et une sensibilité suffisantes, le dommage radiobiologique.

\section{BIBLIOGRAPHIE}

(I) Albaum, H.G. Serum enzymes following whole body radiation in the rabbit. Rad. Res., 12, 186-194, 1960.

(2) Andrews, G.A., Sitrterson, G.W., Kretcmar, A.L. \& Brucer, M. Irradiation accidentelle au centre $Y_{12}$. Diagnostic et traitement des radiolésions aiguës, Genève, 17-21 oct. 1960. O.M.S., Genève, 1964 .

(3) ANGEL, C.R. \& Noonan, T.R. Urinary taurine excretion and the partition of sulfur in four species of mammals after whole body X irradiation. Rad. Res., i 5, 298-306, 1961 .

(4) Bates, T.H., Sмrтh, C.I. \& Sмrтh, H. Radiation induced changes in metabolism of thymine in rat. Nature, 203, 843-844, 1964 .

(5) BeCKer, F.F., Williams, R.B. Jr \& Voogd, J.L. Effect of X irradiation in sublethal to suprelethal dosage in serum glutamic oxalacetic transaminase. Rad. Res., 20, $221-226,1963$.

(6) Berry, H.K., Saenger, E.L., Perry, H., Friedman, B.I., Kereiakes, J.G. \& Scheel, C. Deoxycytidine in urine of human afte1 whole body irradiation. Science, 142, 396-398, 1963.

(7) Bigwood, E.J. \& Soupart, P. $\beta$ amininoisobutyric acid and taurine excretion and plasma levels after local $\mathrm{X}$ ray irradiation in two cancers patients. Biological effects of ionizing radiations at the molecular levels. A.I.E.A., Vienne, 277-286, 1962. 
(8) Boquet, P.L. \& Fromageot, P. Sur l'origine de la taurine excrétée par le rat irradié, B.I.S.T., $93,7-18,1965$.

(9) Castre, W.O. \& Armstrong, W.D. Electrolyte balances following total body X irradiation. Proc. Soc. Exptl. Biol. Med., 90, 56-57, 1955.

(I0) Caster, W.O. \& Armstrong, W.D. Electrolyte metabolism after total body X irradiation. Rad. Res., 5, 189-204, 1956.

(II) Conard, R.A. Cholinesterase activity, weight, water content and pathology of small intestine of rats subjected to X radiation. Am. J. Physiol., 170, 418-425, 1952.

(12) Conard, R.A. Some effects of ionising radiation on the physiology of the gastro-intestinal tract : a review. Rad. Res., 5, 167-188, 1956.

(13) Dalrymple, G.V., Lindsay, I.R. \& Ghidoni, J.J. The effect of $2 \mathrm{MeV}$ X Rays on whole body irradiated primates. S.A.M.-TR-65-9. USAF School of Aerospace Medicine. Brooks Air Force Base, Texas, 1965.

(14) Dalrymple, G.V., Linsday, I.R., Ghidoni, C.B., Kundel, H.L. \& Still, E.T. The effect of massives doses of $32 \mathrm{MeV}$ protons and $60 \mathrm{Co}$ gamma radiation on serum levels of whole body irradiated primates. J. Nucl. Med., 6, 8, 588-593, 1965.

(is) Deanovic, Z., Supek, Z \& Randic, M. Relation ship between the dose of whole body X irradiation and the urinary excretion of $s$-hydroxyindolacetic acid in the rats. Int. J. Rad. Biol., 7, 1, 1-9, 1963.

(16) Drtzes, J. The effect of total body $\mathrm{X}$ irradiation on serum proteins in the hamster. Rad. Res., 5, 694-702, 1962.

(17) Drahovsky, D., Winkler, A. \& SKodA, J. Increased urinary excretion in rats following irradiation. Nature, 20I, 4I I-412, 1964 .

(18) Franzen, F., Gross, H. \& Friedrich, G. Biogene amine in urin und blut von ratten nach subletales ganzkörperbestrahlung. Strablentberapie, 122, 4, 591-594, 1963.

(ig) Franzen, F., Gross, H. \& Tielicke, G. Biogene amine in urin und blut von ratten nach subletaler ganzkörperbestrahlung. Strablentherapie, 120, 598-610, 1963.

(20) Gerber, G., Gertler, P., Altman, K.I. \& Hempelmann, L. Dose dependency of radiation induced creatine excretion in rat urine. Rad. Res., I5, 307-313, 1961.

(2i) Gerber, G., Gerber, G., Kurohara, S., Altman, K.I. \& Hempelmann, L.H. Urinary excretion of several metabolites in persons accidentally exposed to ionizing radiation. Rad. Res., 15, 314-318, 1961.

(22) Gerber, G.B., Gerber, G \& Altman, K.I. The mechanism of radiation induced creatinuria. P.S.E.B.M., $110,797-799,1962$.

(23) Gerber, G.B., Gerber, G., Kosalka, T.R. \& Hempelmannl, H. Creatine metabolism after X radiation. II. Direct and indirect effect of radiation. Rad. Res., 23, 648-652, 1964.

(24) Gerber, G.B. Die Ursachen der Strahlenbedingten Ausscheidung von $\beta$ aminoisobuttersaure und creatin. Rapport Euratom, EUR 591 d, 1964.

(25) GJESSING, E.C. \& W WRREN, S. Effect of radiation on excretion of some of nitrogenous constituents of urine in man. Rad. Res., 15, 276-289, 1961.

(26) Gustafson, G.E. \& Koletsjy, S. Nitrogen metabolism following whole body X radiation. Am. J. Pbysiol., 171, 319-324, 1952.

(27) Hempelmann, L.H. Evaluation de la gravité des radiolésions aiguës. Diagnostics et traitement des radiolésions aiguës. Genève, 17-21 oct. 1960. O.M.S. Genève, 1964.

(28) Hietbrink, B.E., Raymund, A.B., Zins, G.R. \& Dubois, K.P. Enzymatic measurement of the radioprotective activity of chemicals agents. Toxicology and applied pbarmacology, 3 , 3, 267-277, 1961 .

(29) Hugres, L.B. A survey of serum enzymes as a possible index of radiation injury. Los Alamos Scientific Laboratory. Report LA 2167, x-61, 1958.

(30) JAMmet, H.P. Traitement des personnes irradiées lors de l'accident survenu à Vinca avec le réacteur de puissance zéro. Diagnostic et traitement des radiolésions aiguës, Genève, 17-21 oct. 1960, O.M.S., Genève, 1964.

(31) Kay, R.E. \& Entenman, C. Free aminoacids in the tissus and urine of the $\mathrm{X}$ irradiated rat. Fedration Proc., 13, 520, 1954.

(32) KAY, R.E., EARLY, J.C. \& ENTENMAN, C. Increased urinary excretion of taurine and urea by rats after irradiation. Rad. Res., 6, 98-107, 1957. 
(33) KAy, E.R. \& Entenman, C. The effect of multiple exposures and partial body X irradiation on urinary taurine excretion by the rat. Rad. Res., I1, 357-369, 1959.

(34) KrETCH MAr, A.L. An alteration in the excretion of free serine in urine from irradiated humans. Nature, 133, 1809-1810, 1959.

(35) Kusin, A.M. Radiation biochemistry. Israel Program for Scientific Translations, Jerusalem, 1964 .

(36) LANZ, H. \& RUBINI, J.R. Radiobiological significance of $\beta$ aminoisobutyric acid. Report A.E.C.A.T. (40-1), $2731,1964$.

(37) Lipkan, N.F. Elementy Radiatsionniy Biologii i Biokbimii, 45-136, Kiev, 1963.

(38) Lorseleur, J. \& Perrt, M. Augmentation de la bilirubinémie consécutivement à l'irradiation du rat par les rayons X. C.R. Soc. Biol., 1 164-1 165, 1963.

(39) Marblé, G., Frossard, H., Breuil, L. \& Engler, R. Evolution de la glycémie chez le rat après irradiation gamma. Rapport C.E.A. $\mathrm{R} 2922,1965$.

(40) Marblé, G. et Breurl, L. Excrétion urinaire du chlore, du sodium et du potassium chez le rat après irradiation gamma. Non publié.

(4I) Mrichen, F.W. \& Short, A.W. A method for the rapid determination of betaaminoisobutyrique acid (B.A.I.A.B.) in urine of irradiated humans. Int. J. Rad. Biol., 6, 5, 495497, 1963.

(42) Naftalin, L. Further observations on aminoacids changes in plasma and urine following therapeutic irradiation. Int. J. Rad. Biol., 8, 4, 396, 1965 .

(43) Prizek, J., Arient, M., Dienstbier, Z \& SkodA, J. The detection of desoxycytidine in urine as an indicator of cbanges after irradiation. Proceedings of the Second United Nations International Conférence on the Peaceful Uses of Atomic Energy. Rapport P/2498, vol. 22b, 206-207, Genève, 1958.

(44) PEndic, B. Accident à Vinca avec le réacteur de puissance zéro. Diagnostic et traitement des radio-lésions aiguës. O.M.S., Genève, 1964.

(45) PIERucci, O. The effect of whole body radiation on the acid desoxyribonuclease (DNase II) level of spleen of $\mathrm{Ha} / \mathrm{ICR}$ swiss mice. Radiation Research Society, Twelfth annual meeting, Miami Beach, Florida, May 18-20, 1964.

(46) Rdojicrc, B., Hadjukovic, S. \& Anric, M. Surveillance des personnes irradiées lors de l'accident survenu à Vinca avec le réacteur de puissance zéro. Diagnostic et traitement des radiolésions aiguës. O.M.S., Genève, 1964.

(47) RANDIC, M. \& SUPEK, Z. Urinary excretion of s-hydroxyindolacetic acid after a single whole body irradiation in normal and adrenalectomized rats. Int. J. Rad. Biol., 4, 2, I 5 I-I 53 , 196r.

(48) RAppoport, D.A. \& Fritz, R.D. Influence of total body X irradiation on nucleoside phosphorylase in rat erythrocytes. Rad. Res., $21,5-15,1964$.

(49) Rubini, J.R., Cronkrte, E.P., Bond, V.P. \& Fliedner, T.M. Urinary excretion of betaaminoisobutyric acdi (BAIBA) in irradiated humans beings. P.S.E.B.M., 100, 130-133, 1959.

(so) SAEngeER, E.L. Metabolic changes in bumans following irradiation. D.A.S.A., 1422, 1963.

(s I S Sirman, T.L. Cas d'irradiation mortelle par surexposition accidentelle massive à des neutrons et à des rayons gammas. Diagnostic et traitement des radiolésions aiguës. O.M.S., 1964 .

(52) SchöN, H., Srrzmann, F.C. \& Barth, G. Das Verhalten der Brenztraubensäure I (+) milchsäure und alphaketoglutasäure im serum bei therapeutischen anwerdung von röntgenstrahlen. Strablentberapie, 105, 585-591,1958.

(53) ScoppA, P. \& Gerbaulet, K. Escrezione di glucurodini urinari nel ratto irradiato. Rapport Euratom. EUR 2638 i, 1966.

(54) Skalka, M. \& Matrasova, J. The effect of low radiation doses on the release of deoxyribopolynucleotides in heamapoietic and lymphatic tissues. Int. J. Rad. Biol., 7, I, 41-44, 1963.

(55) Sмrтh, H., Bates, T.H. \& SMrrh, C.J. Excretion of $\beta$ aminoisobutyric acid as an index of radiation exposure. Int. J. Rad. Biol., 8, 3, 263-270, 1964.

(56) SMrrt, H. \& BATES, T.H. An assessment of those metabolites considered to be of value in the diagnosis of exposure to radiation. Personnel dosimetry for accidental high-level exposure to external and internal radiation. A.I.E.A., Vienne, 199-215, 1965.

(57) StrRn, D.N. \& Strm, E.M. Sources of excess taurine excreted in rats following whole body irradiation. P.S.E.B.M., ror, $125-158,1958$. 
(58) Tomin, Z.L., ANDRI, L. \& CAprotri, M. Il comportamento della colinesterasi nel sangue e nei tessuti di ratti sottoposti a radiazoni ionizzanti. Folia Medica, XLVI, 3, 189-194, 1963.

(s9) WALD, N. \& Tномл, G.E. Jr. Radiation accidents : medicals aspects of neutron and gamma ray exposures. O.R.N.L., 2748, part. B, 1961.

(6o) Watson, G.M. The origine of taurine excreted in the urine after whole body irradiation. Int. J. Rad. Biol., 5, 1, 79-83, 1961 .

(6i) Williams, C.M., Krise, G.M., Anderson, D.R. \& Dowben, R.M. Post-irradiation creatinuria in the rat. Rad. Res., 7, 176-183, 1957.

(62) Winkler, C. \& Paschke, G. Protein content and composition of rat serum as related to amount of whole body X irradiation. Rad. Res., 5, 156-161, 1956. 
